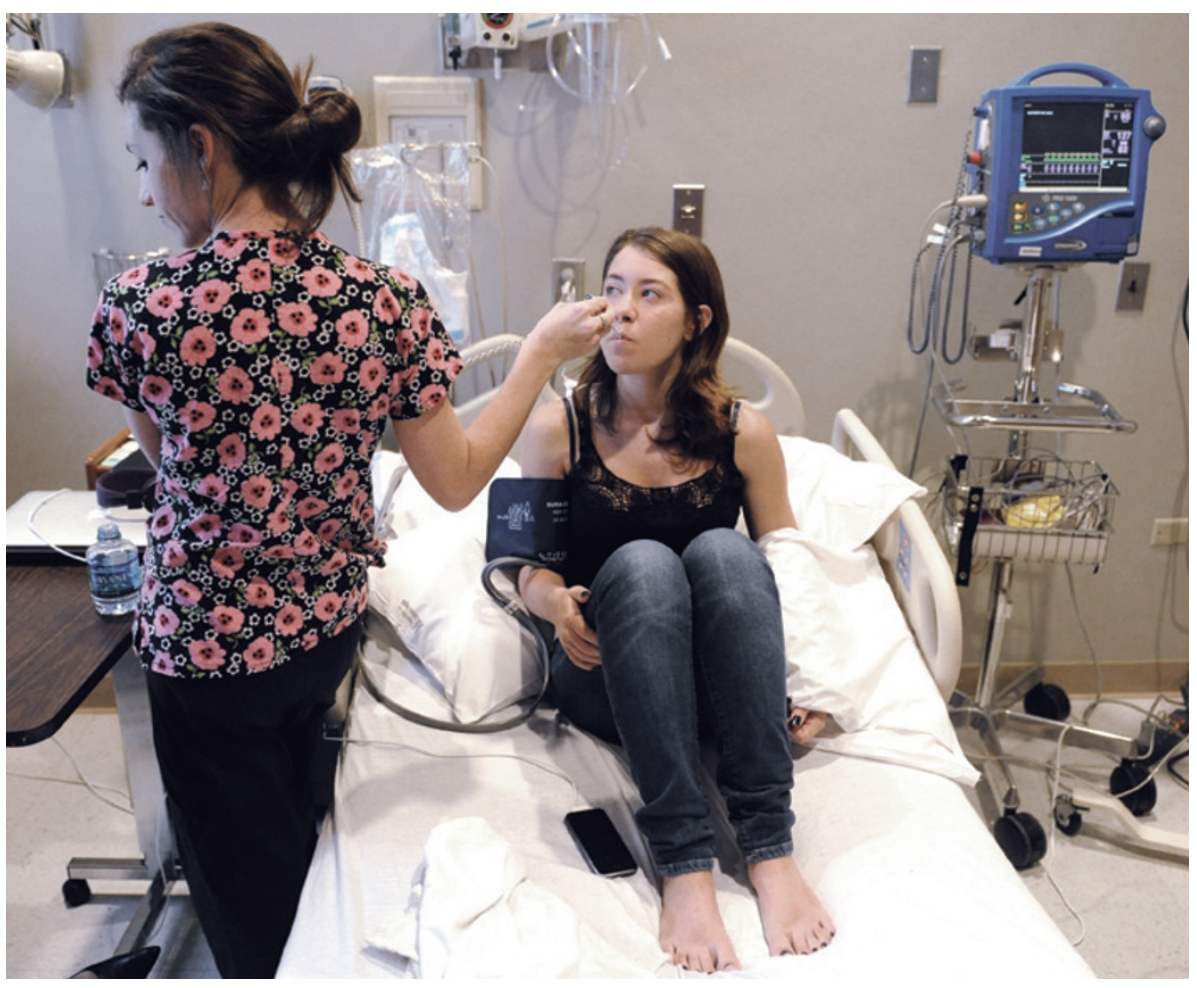

Kerby Bennett is a participant in the NIH's TrialNet diabetes prevention study.

IMMUNOMODULATORS

\title{
Cell savers
}

\section{In type 1 diabetes, the immune system goes haywire and depletes insulin-producing cells. Drugs that interfere with this process could one day reverse the disease's course.}

\section{BY SARAH DEWEERDT}

$\mathrm{H}$ eart attack, stroke, aneurysm: each is a potentially fatal event. Most people probably wouldn't consider type 1 diabetes to be as urgent, but at least one researcher argues that it is.

Jean-Francois Bach, an immunologist at the University of Paris-Descartes, argues that type 1 diabetes (T1D) should be considered a "medical emergency", and that the goal of treatment should be to reverse the disease as soon as possible after diagnosis.

T1D occurs when the immune system mistakenly attacks beta cells - the insulinproducing cells in the pancreas. The assault takes years before the disease manifests, but only one-third of beta cells survive in most patients by the time diabetes is diagnosed, so it is critical to save those beta cells that remain. If the immune attack could be halted and those remaining cells could be preserved, scientists believe that they could be sufficient to produce most of the body's insulin on their own.
Over the past decade, numerous clinical trials have tried to use immune-modifying drugs, many borrowed from the treatment of other autoimmune diseases, to try to save beta cells. So far, none have worked especially well. And because the disease is most often diagnosed in children and young adults, any effective treatment would have to be tolerated over the course of many years. But researchers are continuing to hone the dosage, timing and perhaps combination of drugs to a therapy that works.

\section{HIGH HOPES}

In the 1980s, studies demonstrated that suppressing the immune system of people recently diagnosed with T1D reduced their insulin dependence and provided persuasive evidence that T1D is an autoimmune disease. "Back then it wasn't so clear," says immunologist Jeffrey Bluestone of the University of California, San Francisco.

The early immunomodulators, such as cyclosporine and antithymocyte globulin, were blunt instruments, targeting not just the immune cells responsible for killing the beta cells, but other parts of the immune system as well. The drugs were too toxic for patients to take for extended periods, and any protective effect failed to persist after treatment. But even this limited effectiveness was enough to spur researchers to look for more specific immune modulators that would affect the precise mechanisms of beta-cell destruction in diabetes.

Much attention has focused on T cells, thought to be behind the targeted killing of beta cells in T1D. Antibodies against CD3, a receptor found on T cells, can prevent or even permanently reverse diabetes in non-obese diabetic (NOD) mice and other mouse models of T1D. "What was exciting in the mouse model was that we gave short-term dosing and we got long-term effects," says Bluestone. He and other researchers hope that anti-CD3 might attenuate the immune system rather than switch it off with anti-rejection drugs.

Two different anti-CD3 antibody drugs, teplizumab and otelixizumab, have been tested in humans. Studies in Europe and the United States showed that a short course of treatment - two weeks or less - in people recently diagnosed with diabetes can improve beta-cell function for as long as five years.

In contrast to the mouse results, the effect in humans is temporary; eventually, beta-cell depletion begins again and the disease progresses. A bigger setback came in 2011, with the results of two large, phase III clinical trials of these agents on recently diagnosed patients. Although the design and endpoints of the studies were slightly different, both showed that the anti-CD3 approach did not improve on standard insulin therapy after one year ${ }^{1}$.

That's not an unfamiliar result. Indeed, Bluestone says, showing improvement over standard therapy is "really tough in the first year after diagnosis". That's because insulin formulations and delivery systems are now so good that most patients can easily keep their diabetes well controlled at first. Comparing any immunosuppression strategies to insulin treatment has been the undoing of a number of other drug treatments, he says.

The same story of high hopes dashed in phase III trials unfolded for glutamic acid decarboxylase (GAD), a molecule normally found in the pancreas and one of the antigens targeted for destruction in the immune attack. Delivering GAD in a different form might help the immune system regard the molecule as friend rather than foe. But a European study found that injections of GAD failed to either stem the loss of beta-cell function or improve diabetes control over the course of 15 months in recently diagnosed T1D patients ${ }^{2}$. A similar trial in the United States was halted because GAD didn't seem to be effective.

Jay Skyler, chair of Type 1 Diabetes TrialNet, a National Institutes of Health-funded research consortium in the United States, says that the problem with GAD might have been its delivery. Animal studies showed some promise when the molecule was administered orally, nasally or by 
injection into the abdominal cavity, but both the US and European clinical trials used an injection under the skin. "So I personally am not ready to give up on GAD," says Skyler, who is also a professor of medicine and paediatrics at the University of Miami in Florida. "I want to see more data."

There is some optimism about other antigens, such as insulin and its precursor, proinsulin, which might form the basis of other immunemodulating therapies. This approach offers the advantage of specificity: rather than altering the function of whole classes of immune cells, which might render a patient susceptible to infection, antigen therapies affect only activated $T$ cells that attack beta cells. This precision is "a very important safety net", says Chantal Mathieu, an endocrinologist at the Catholic University of Leuven in Belgium.

\section{DIVERSE TWEAKS}

Mathieu is investigating other, gentler strategies for immune modulation. She is coordinator of Natural Immunomodulators as Novel Immunotherapies for Type 1 Diabetes (NAIMIT), an EU-funded project. For example, Mathieu's group has previously shown that vitamin $\mathrm{D}$ can prevent diabetes in NOD mice. "The problem is that the doses we needed were huge," she says — equivalent to 1,000 times what could be used in humans. Such massive amounts of vitamin D can cause heart arrhythmias, kidney stones and dangerously high levels of calcium.

But researchers have not written off vitamin D just yet. A team led by diabetes and immunology specialist Bart Roep at Leiden University in the Netherlands showed that when dendritic cells (a type of immune cell) mature in vitro in the presence of vitamin $\mathrm{D}$, they dampen $\mathrm{T}$-cell responses. "This is a very elegant way of exploiting the immune-modulatory effect of vitamin D," Mathieu says. The team hopes to begin human studies, infusing the dendritic cells back into T1D patients, sometime in 2012.

Another possible immunological tweak is to block costimulation - the final stage of T-cell activation. A TrialNet study taking this approach enrolled 77 people with recently diagnosed diabetes. They each received 27 infusions of abatacept, a costimulation inhibitor used in rheumatoid arthritis, over 2 years. Results were

"Immunotherapy received abatacepthad could extend better beta-cell funcbeyond

treatment to

become the basis

for diabetes prevention." tion than the control group $^{3}$. However, the drug had its strongest effects during the first 6 months of treatment. After that, the abatacept group lost beta-cell mass and function at the same rate as controls.

TrialNet researchers found similar results in a study targeting B cells, which help T cells recognize antigens. They administered four doses of rituximab, an antibody against the CD20 receptor found on B cells, to 57 people with recently

\section{IMMUNOTHERAPY} The object in each case is to prevent the immune system (T cells) from attacking the
beta cells in the pancreatic islet (shown on the right).

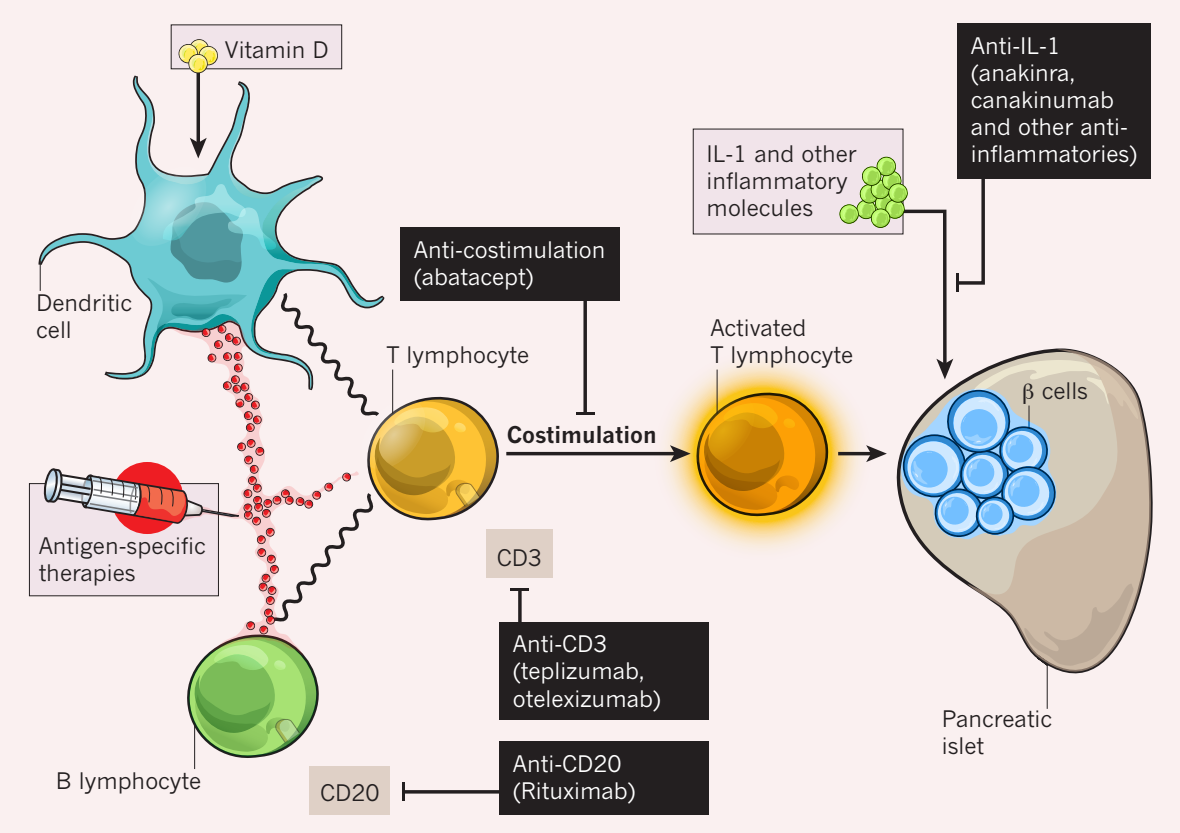

diagnosed diabetes. A year later, those who had received rituximab had better beta-cell function and required less insulin than controls ${ }^{4}$. But again, the effects were most dramatic early on, and by 6 months the rituximab group were also losing beta cells. "These drugs all seem to have similar effects, and they all seem to have effects only during this certain window of time - within that first 3-6 months," says Carla Greenbaum, director of the diabetes programme at Benaroya Research Institute in Seattle, Washington, and vice-chair of TrialNet.

\section{INNATE INTERPLAY}

This research cul-de-sac has prompted researchers to explore more than just the antigen-specific responses of $\mathrm{B}$ and $\mathrm{T}$ cells (that is, adaptive immunity) - and to look at the non-specific, or innate, immune responses such as inflammation, as well. As early as the mid-1980s, endocrinologist Mandrup-Poulsen, now at the University of Copenhagen in Denmark, showed that one molecule involved in innate immunity - the pro-inflammatory interleukin-1 (IL-1) - can kill insulin-producing beta cells. The recent revival of interest in innate immunity has prompted further study. In 2011, a preliminary study found that 15 children recently diagnosed with T1D who were given a 28-day course of the IL-1 blocker anakinra needed less insulin 4 months after diagnosis than did controls ${ }^{5}$.

Two phase II trials of IL-1 blockers in T1D are just wrapping up. A European study headed by Mandrup-Poulsen also uses anakinra; a TrialNet study in the United States uses a similar drug called canakinumab. Both groups plan to announce the results of their studies at the
American Diabetes Association meeting in Philadelphia, Pennsylvania, June 2012. Even if these studies are negative, Mandrup-Poulsen argues, researchers should consider investigating IL-1 blockers as part of combination therapy.

In fact, a recent mouse study provides support for such a strategy. Researchers found that combining anti-CD3 antibody with anakinra - at doses too low for either drug to work alone permanently reversed diabetes in NOD mice ${ }^{6}$. "This is really exciting because it would indicate that if you titrate these two immune modulatory agents you may obtain very potent effects on the disease process," says Mandrup-Poulsen, a coauthor of the study's report.

The impact of immunotherapy could extend beyond treatment. It might also become the basis for diabetes prevention, because the immunerelated destruction of beta cells is thought to begin several years before diabetes becomes clinically apparent. "In theory, stopping the immune attack before it's fully blown ought to be more effective than stopping it when everything is underway," says Skyler. In other words, the best strategy for dealing with an immune emergency would be to prevent it altogether.

Sarah DeWeerdt is a science writer based in Seattle, Washington.

1 Sherry, N. et al. Lancet 378, 487-497(2011).

2. Ludvigsson, J. etal. N. Engl. J. Med. 366, 433-442 (2012),

3. Orban, T. et al. Lancet 378, 412-419 (2011).

4. Pescovitz, M. et al. N. Engl. J. Med. 361, 2143-2152 (2009).

5. Sumpter, K. M. et al. Pediatric Diabetes 12, 656-667 (2011).

6. Ablamunits, V. et al. Diabetes 61, 145-154 (2012). 